\title{
Linearizability of the perturbed Burgers equation
}

\author{
R. A. Kraenkel, J. G. Pereira, and E. C. de Rey Neto \\ Instituto de Física Teórica, Universidade Estadual Paulista, Rua Pamplona 145, 01405-900 São Paulo, Brazil
}

(Received 7 August 1997; revised manuscript received 18 November 1997)

\begin{abstract}
We show in this report that the perturbed Burgers equation $u_{t}=2 u u_{x}+u_{x x}+\epsilon\left(3 \alpha_{1} u^{2} u_{x}+3 \alpha_{2} u u_{x x}\right.$ $\left.+3 \alpha_{3} u_{x}^{2}+\alpha_{4} u_{x x x}\right)$ is equivalent, through a near-identity transformation and up to $O(\epsilon)$, to a linearizable equation if the condition $3 \alpha_{1}-3 \alpha_{3}-\frac{3}{2} \alpha_{2}+\frac{3}{2} \alpha_{4}=0$ is satisfied. In the case this condition is not fulfilled, a normal form for the equation under consideration is given. We show, furthermore, that nonlinearizable cases lead to perturbative expansions with secular-type behavior. Then, to illustrate our results, we make a linearizability analysis of the equations governing the dynamics of a one-dimensional gas. [S1063-651X(98)01908-4]
\end{abstract}

PACS number(s): 03.40.Kf

\section{INTRODUCTION}

The object of this paper is the perturbed Burgers equation

$u_{t}=2 u u_{x}+u_{x x}+\epsilon\left(3 \alpha_{1} u^{2} u_{x}+3 \alpha_{2} u u_{x x}+3 \alpha_{3} u_{x}^{2}+\alpha_{4} u_{x x x}\right)$,

where $\alpha_{i}$ are constants, $\epsilon \ll 1$ is a perturbative parameter, and subscripts denote partial differentiation. It appears in the long-wave, small-amplitude limit of extended systems dominated by dissipation, but where dispersion is also present at a higher order. More precisely, those systems described by equations whose linear part admits a dispersion relation of the form

$$
\Omega(k)=a_{3} k^{3}+a_{5} k^{5}+\cdots+\imath\left(b_{2} k^{2}+b_{4} k^{4}+\cdots\right),
$$

with $a_{i}$ and $b_{i}$ real constants. For example, Eq. (1) appears in the description of gas dynamics [1], and in certain cases of free-surface motion of waves in heated fluids [2]. More important, however, is the fact that the terms appearing at order $\epsilon$ are the only ones allowed if Eq. (1) is obtained from longwave perturbation theory, and no constants are allowed to scale with $\epsilon$. In this sense, it has the universality characteristics, much in the same way as the equations discussed by Calogero [3].

When the $O(\epsilon)$ terms are discarded, we have simply a Burgers equation, which is an equation linearizable through a Hopf-Cole transformation [4]. It is, thus, a natural question to know when Eq. (1) is also linearizable. Put in this way, the answer is that it is linearizable if $\alpha_{1}=\alpha_{2}=\alpha_{3}=\alpha_{4}$, in which case the equation is reduced to the sum of Burgers with the first higher-order equation of the Burgers hierarchy [5]. We notice in passing that the latter is also linearizable by the same Hopf-Cole transformation that linearizes the Burgers equation. However, we can put the question on a more general setting by introducing the idea of near identity transformation [6], that is, a transformation $u \rightarrow w$ of the form

$$
u=w+\epsilon \phi(w) .
$$

If we apply such a transformation to Eq. (1), we may look for functions $\phi(w)$ such that the transformed equation reads:

$$
\begin{aligned}
w_{t}= & 2 w w_{x}+w_{x x}+\epsilon \lambda\left(3 w^{2} w_{x}+3 w w_{x x}\right. \\
& \left.+3 w_{x}^{2}+w_{x x x}\right)+O\left(\boldsymbol{\epsilon}^{2}\right),
\end{aligned}
$$

for some $\lambda \in \mathbb{R}$. If such a $\phi(w)$ exists, we say that Eq. (1) and Eq. (4) are equivalent up to $O(\epsilon)$, and this is the equivalence that is physically relevant, as long as perturbation theory is concerned. As Eq. (4) is linearizable, so is Eq. (1) up to $O(\epsilon)$. The fundamental issue here is thus to determine the conditions for the existence of a near identity transformation [that is, $\phi(w)$ ] ensuring the equivalence, up to $O(\epsilon)$, of Eqs. (1) and (4). This is the question we will address in this paper, and an answer will be given in terms of a condition on the parameters $\alpha_{1}, \alpha_{2}, \alpha_{3}$, and $\alpha_{4}$.

This kind of equivalence is usually introduced in the context of normal form analysis of ordinary differential equations, and has been discussed for dispersive partial differential equations in $[7,8]$. When the lowest order, in the longwavelength limit, of such system is described by the Korteweg-de Vries equation $(\mathrm{KdV})$, there exists always a near-identity transformation that makes the $O(\epsilon)$ perturbations integrable. Indeed, in Ref. [8], it has been shown that, by introducing a $\phi(w)$ depending explicitly on $x$, one can completely remove the $O(\epsilon)$ corrections. However, obstacles to integrability appear at $O\left(\epsilon^{2}\right)[7,9,10]$. For a similar analysis in the case of the nonlinear Schrödinger equation, the reader is addressed to [11].

We will show that, in the case of Eq. (1), obstacles to linearizability appear already at $O(\epsilon)$. We mean by this that, in general, Eq. (1) is not equivalent to Eq. (4). The condition for the equivalence will be shown to be

$$
3 \alpha_{1}-3 \alpha_{3}-\frac{3}{2} \alpha_{2}+\frac{3}{2} \alpha_{4}=0 \text {. }
$$

Furthermore, in the case where condition (5) is not satisfied, we find a normal form for Eq. (1). Finally, as an illustration, we make a linearizability analysis of the equations governing the dynamics of a one-dimensional gas, and we show that, already at order $O(\epsilon)$, these equations cannot be linearized.

\section{LINEARIZABILITY ANALYSIS}

Let us then implement the ideas exposed above. We want to insert Eq. (3) into Eq. (1), discard all $O\left(\epsilon^{2}\right)$ terms, and compare the result with Eq. (4). To do so, we have to specify 
the possible form of $\phi(w)$. They ought to be such as to generate, at $O(\epsilon)$, terms of the form $w^{2} w_{x}, w w_{x x}, w_{x}^{2}$, and $w_{x x x}$. The allowable terms turn out to be $w_{x}, w^{2}$, and $w_{x} \partial^{-1} w$, where $\partial^{-1}$ means integration in $x$. Thus the general form of $\phi(w)$ is

$$
\phi(w)=\alpha w_{x}+\beta w^{2}+\gamma w_{x} \partial^{-1} w,
$$

where $\alpha, \beta$, and $\gamma$ are constants to be determined.

We introduce now the following useful notations:

$$
B(u)=2 u u_{x}+u_{x x},
$$

and

$$
\Theta(u)=3 \alpha_{1} u^{2} u_{x}+3 \alpha_{2} u u_{x x}+3 \alpha_{3} u_{x}^{2}+\alpha_{4} u_{x x x} .
$$

This makes Eq. (1) read

$$
u_{t}=B(u)+\epsilon \Theta(u) .
$$

The transformation (3) changes Eq. (9) to an equation in $w$, given by

$$
w_{t}=B(w)+\boldsymbol{\epsilon}\{\Theta(w)+[B(w), \phi(w)]\}+O\left(\epsilon^{2}\right),
$$

where

$$
[B(w), \phi(w)]=\frac{\delta B}{\delta w} \phi-\frac{\delta \phi}{\delta w} B .
$$

In order to obtain the transformed equation, we have thus to calculate the commutator $[B(w), \phi(w)]$, which is the tedious part of our task. After performing that calculation, we get

$$
[B(w), \phi(w)]=2 \beta w_{x}^{2}+2 \gamma w w_{x x}+(2 \beta+\gamma) w^{2} w_{x} .
$$

Inserting this into Eq. (10), the transformed equation reads

$$
\begin{aligned}
w_{t}= & B(w)+\epsilon\left[\left(2 \beta+\gamma+3 \alpha_{1}\right) w^{2} w_{x}+\left(2 \gamma+3 \alpha_{2}\right) w w_{x x}\right. \\
& \left.+\left(2 \beta+3 \alpha_{3}\right) w_{x}^{2}+\alpha_{4} w_{x x x}\right] .
\end{aligned}
$$

If we now require Eq. (12) to be of the form given by Eq. (4), we have to take $\lambda=\alpha_{4}$, and the following conditions must be satisfied:

$$
\begin{gathered}
2 \beta=3 \alpha_{4}-3 \alpha_{3}, \\
2 \gamma=3 \alpha_{4}-3 \alpha_{2}, \\
2 \beta+\gamma=3 \alpha_{4}-3 \alpha_{1} .
\end{gathered}
$$

Clearly, this system of equations is not always solvable. The solubility condition is

$$
3 \alpha_{1}-3 \alpha_{3}-\frac{3}{2} \alpha_{2}+\frac{3}{2} \alpha_{4}=0,
$$

in which case $\beta=\frac{3}{2}\left(\alpha_{4}-\alpha_{3}\right)$ and $\gamma=\frac{3}{2}\left(\alpha_{4}-\alpha_{2}\right)$. Note that $\alpha$ is left undetermined. Condition (16) is thus the condition that must be satisfied in order to make Eq. (1) equivalent, up to $O(\epsilon)$, to Eq. (4).

Suppose now that Eq. (16) is not satisfied. The general form of the transformed equation is given by Eq. (12). The
$O(\epsilon)$ terms can be written as the sum of a linearizable term proportional to $\alpha_{4} F_{3}(w)$, with

$$
F_{3}(w)=3 w^{2} w_{x}+3 w w_{x x}+3 w_{x}^{2}+w_{x x x},
$$

plus a term $Z(w)$ representing the obstacle to linearizability, that is,

$$
w_{t}=B(w)+\epsilon \alpha_{4} F_{3}(w)+\epsilon Z(w),
$$

where

$$
\begin{aligned}
Z(w)= & \left(2 \beta+3 \alpha_{3}-3 \alpha_{4}\right) w_{x}^{2}+\left(2 \gamma+3 \alpha_{2}-3 \alpha_{4}\right) w w_{x x} \\
& +\left(2 \beta+\gamma+3 \alpha_{1}-3 \alpha_{4}\right) w^{2} w_{x} .
\end{aligned}
$$

If we call each of the coefficients appearing in the obstacle respectively by $\mu_{1}, \mu_{2}$, and $\mu_{3}$, and if we further introduce $\nu_{i}$ through $\mu_{i}=\mu \nu_{i}$, with $\mu=3 \alpha_{1}-3 \alpha_{3}-\frac{3}{2} \alpha_{2}+\frac{3}{2} \alpha_{4}$, then we may write out the normal form of Eq. (1) as

$$
w_{t}=B(w)+\epsilon \alpha_{4} F_{3}(w)+\epsilon \mu\left(\nu_{1} w_{x}^{2}+\nu_{2} w w_{x x}+\nu_{3} w^{2} w_{x}\right),
$$

where $\nu_{i}$ are arbitrary constants satisfying

$$
\nu_{1}-\nu_{3}+\frac{\nu_{2}}{2}=-1
$$

Equation (20) encompasses the main results of this letter: for $\mu=0$ we have a linearizable equation, and for $\mu \neq 0$ it gives the general form to which Eq. (1) is equivalent up to $O(\epsilon)$.

\section{HOPF-COLE TRANSFORMATION AND PERTURBATIVE EXPANSIONS}

A further simplification can be achieved if, instead of Eq. (6), we introduce, following Ref. [8], a more general transformation, given by

$$
\phi(w)=\alpha w_{x}+\beta w^{2}+\gamma w_{x} \partial^{-1} w+\nu x\left(w_{x x}+2 w w_{x}\right)
$$

with $\nu$ a constant. This leads to the following normal form:

$$
\begin{aligned}
w_{t}= & B(w)+\epsilon\left(\alpha_{4}+2 \nu\right) F_{3}(w)+\epsilon \mu\left(\nu_{1} w_{x}^{2}+\nu_{2} w w_{x x}\right. \\
& \left.+\nu_{3} w^{2} w_{x}\right),
\end{aligned}
$$

where $\mu$ is not modified, relation (21) is still valid, but the coefficients $\nu_{1}$ and $\nu_{3}$ have new expressions in terms of the parameters defining the transformations. Explicitly, we have

$$
\begin{gathered}
\nu_{1}=\mu^{-1}\left(2 \beta+3 \alpha_{3}-3 \alpha_{4}-2 \nu\right), \\
\nu_{3}=\mu^{-1}\left(2 \beta+\gamma+3 \alpha_{1}-3 \alpha_{4}-2 \nu\right) .
\end{gathered}
$$

The meaning of this result is the following: the new transformation generated by Eq. (22) does not have any influence on the linearizability up to $O(\epsilon)$ of Eq. (1), that is, it does not alter condition (16). But, it makes it possible to further simplify the normal form by taking $\nu=\alpha_{4} / 2$. This completely eliminates the $F_{3}(w)$ term from Eq. (23). We are thus lead to the study of the equation 


$$
w_{t}=B(w)+\epsilon \mu\left(\nu_{1} w_{x}^{2}+\nu_{2} w w_{x x}+\nu_{3} w^{2} w_{x}\right)
$$

Let us now proceed to make a Hopf-Cole transformation given by

$$
w=\frac{f_{x}}{f} .
$$

Inserting in Eq. (26) we get

$$
f_{x t} f-f_{x} f_{t}=f_{x x x} f-f_{x} f_{x x}+\epsilon f^{2} Z(f),
$$

with $Z(f)=\mu\left(\nu_{1} w_{x}^{2}+\nu_{2} w w_{x x}+\nu_{3} w^{2} w_{x}\right)$. This means that, in general, we do not have a linear equation anymore, as would be the case if $Z(f) \equiv 0$. Let us try to perform a perturbative expansion for $f$ by setting

$$
f=f_{0}+\epsilon f_{1}+\cdots .
$$

We get, by comparing order by order in Eq. (28),

$$
\begin{gathered}
L\left(f_{0}\right)=0, \\
f_{0}\left[L\left(f_{1}\right)\right]_{x}-f_{0 x} L\left(f_{1}\right)=f_{0}^{2} Z\left(f_{0}\right),
\end{gathered}
$$

where $L(\cdot)=\partial_{t}-\partial_{x x}$. As a consequence of these equations we see that, if we have a solution of Eq. (30) such that there exist constants $\nu_{i}$ satisfying the constraint (21), for which $Z\left(f_{0}\right)=0$, then we may take $f_{1} \equiv 0$. This means that $f_{0}$ is an exact solution to Eq. (28). This is the case, for instance, for the shock-type solution

$$
f_{0}=1+e^{\Theta(k)},
$$

with $\Theta(k)=k x+k^{2} t$. If we take $\nu_{1}=-\nu_{2}=-\nu_{3}=-\frac{2}{3}$, then $Z\left(f_{0}\right)=0$. However, this is an exceptional case. To analyze more general situations, we first note that Eq. (31) can be seen as a first-order linear nonhomogeneous equation for $L\left(f_{1}\right)$. Solving it, we get

$$
L\left(f_{1}\right)=f_{0} \int d \xi Z\left(f_{0}\right)
$$

which is a diffusion equation with a space and time dependent source term. It is thus clear that, depending on the "intensity" of the source, Eq. (33) may not have always bounded solutions. This is a signal that such perturbative expansions are not uniformly valid. This is not surprising, as such is also the case for the perturbed $\mathrm{KdV}$ equation [7]. It is useful to have a more explicit example. Let us take

$$
f_{0}=1+e^{\Theta\left(k_{1}\right)}+e^{\Theta\left(k_{2}\right)} .
$$

It is not difficult to see that there do not exist $\nu_{i}$ allowing $Z\left(f_{0}\right)=0$. We take then the same $\nu_{i}$ as in the shock solution (32). We come to the following expression for $Z\left(f_{0}\right)$ :

$$
Z\left(f_{0}\right)=\frac{2 \mu}{3 f_{0}^{2}}\left[k_{1} k_{2}\left(k_{1}-k_{2}\right)^{2} e^{\Theta\left(k_{1}\right)+\Theta\left(k_{2}\right)}\right] .
$$

A general solution for Eq. (33) is not possible in this case. Let us introduce a further approximation by putting $k_{1}-k_{2}$ $=\Delta k$, and let us compute $Z\left(f_{0}\right)$ to the lowest order in $\Delta k$. Clearly, this gives

$$
Z\left(f_{0}\right)=\frac{2 \mu(\Delta k)^{2} k_{1}^{2}}{3}\left[\frac{e^{2 \Theta\left(k_{1}\right)}}{F^{2}}\right]+O\left(\Delta k^{3}\right),
$$

where $F=\left.f_{0}\right|_{k_{1}=k_{2}}=1+2 e^{\Theta\left(k_{1}\right)}$. We can now explicitly integrate this expression, yielding the following equation for $f_{1}$ :

$$
L\left(f_{1}\right)=(\Delta k)^{2} \frac{k_{1} \mu}{6}[1+F \ln F] .
$$

Let us now look for a solution to Eq. (37) by supposing that $f_{1}=f_{1}\left(\Theta\left(k_{1}\right)\right)$. This implies the following ordinary differential equation for $f_{1}$ :

$$
f_{1}^{\prime}-f_{1}^{\prime \prime}=\frac{(\Delta k)^{2} \mu}{6 k_{1}}[1+F \ln F] .
$$

Instead of solving this equation directly, let us notice the following point. The variable that represents the first-order correction to $w_{0}$ is $\partial_{x}\left(f_{1} / f_{0}\right)$ [with $w_{0}=\left(f_{0}\right)_{x} / f_{0}$ ]. Remembering that $F=\left.f_{0}\right|_{k_{1}=k_{2}}$ we are naturally led to define $f_{1}=$ $F g\left(\Theta\left(k_{1}\right)\right)$. Inserting this definition in Eq. (38), we come to an equation for $g^{\prime}$ that is exactly the relevant variable, which reads:

$$
\begin{aligned}
g^{\prime \prime} & \left(1+2 e^{\Theta\left(k_{1}\right)}\right)+g^{\prime}\left(1-2 e^{\Theta\left(k_{1}\right)}\right) \\
& =\frac{(\Delta k)^{2} \mu}{6 k_{1}}\left[1+\left(1+2 e^{\Theta\left(k_{1}\right)}\right) \ln \left(1+2 e^{\Theta\left(k_{1}\right)}\right)\right] .
\end{aligned}
$$

The solution to this equation for $g^{\prime}$ can be found by elementary methods, and is given by

$$
\begin{aligned}
g^{\prime}(\Theta)= & -\frac{(\Delta k)^{2} \mu}{6 k_{1}} e^{-\Theta\left(k_{1}\right)\left\{\frac{1}{4}+\frac{1}{2}\left(1+2 e^{\Theta\left(k_{1}\right)}\right)\right.} \\
& \left.\times\left[1+\ln \left(1+2 e^{\Theta\left(k_{1}\right)}\right)\right]\right\} .
\end{aligned}
$$

This solution diverges linearly with $\Theta\left(k_{1}\right)$ for $\Theta\left(k_{1}\right) \rightarrow \infty$, indicating that the perturbative expansion fails. This is quite similar to the secular behavior present in many cases of perturbative expansions for differential equations [12]. We should notice, however, that this is not a usual secular term coming from linear resonances that are eliminated by procedures like the multiple-scale method. Finally, we note that the transformation given by Eq. (22) is not essential for the derivation of the above results, which could be obtained without making use of it.

\section{GAS DYNAMICS}

Let us consider the equations governing the dynamics of a one-dimensional gas [1]

$$
\begin{gathered}
\rho_{t}+(\rho u)_{x}=0, \\
(\rho u)_{t}+\left[\rho u^{2}+P-\mu u_{x}\right]_{x}=0,
\end{gathered}
$$


where $\rho(x, t)$ is the density, $u(x, t)$ is the velocity, $\mu$ is the viscosity, and

$$
P=A\left(\frac{\rho}{\rho_{0}}\right)^{\gamma}
$$

is the pressure, with $\gamma=\left(c_{p} / c_{v}\right)$ the ratio of specific heats, and $A$ a proportionality constant. In order to study its longwavelength, small-amplitude limit, we define slow space and time variables,

$$
\begin{gathered}
\xi=\epsilon(x-c t), \\
\tau=\epsilon^{2} t,
\end{gathered}
$$

and scale the original (primed) density and velocity fields according to

$$
\begin{gathered}
\rho^{\prime}=\rho_{0}+\epsilon \rho, \\
u^{\prime}=\epsilon u .
\end{gathered}
$$

In terms of these new variables, Eqs. (41) and (42) become

$$
\begin{gathered}
\rho_{0} u_{\xi}-c \rho_{\xi}+\epsilon\left[\rho_{\tau}+(u \rho)_{\xi}\right]=0 \\
\frac{A \gamma}{\rho_{0}} \rho_{\xi}-c \rho_{0} u_{\xi}+\epsilon\left[-c(u \rho)_{\xi}+\rho_{0} u_{\tau}+\rho_{0} u_{\tau}+\rho_{0}\left(u^{2}\right)_{\xi}\right. \\
\left.-\mu u_{\xi \xi}\right]+\epsilon^{2}\left[(u \rho)_{\tau}+\left(\rho u^{2}\right)_{\xi}\right]=0 .
\end{gathered}
$$

Moreover, as a compatibility condition at $O\left(\epsilon^{0}\right)$, we have to set

$$
c^{2}=\frac{A \gamma}{\rho_{0}} .
$$

Now, from Eq. (47) we obtain

$$
\rho_{\xi}=\frac{\rho_{0}}{c} u_{\xi}+\frac{\epsilon}{c}\left[\rho_{\tau}+(u \rho)_{\xi}\right]+O\left(\epsilon^{2}\right)
$$

or

$$
\rho=\frac{\rho_{0}}{c}\left[u+\frac{\epsilon}{c}\left(u^{2}-\partial^{-1}\left(u_{\tau}\right)\right)\right]+O\left(\epsilon^{2}\right),
$$

with $\partial^{-1}$ indicating an integration in the $\xi$ coordinate. Substituting in Eq. (48), and using the resulting equation into itself, we are led to

$$
\begin{aligned}
u_{\tau}= & -u u_{\xi}+\frac{\mu}{2 \rho_{0}} u_{\xi \xi}+\epsilon\left[\frac{1}{c} u^{2} u_{\xi}-\frac{3 \mu}{2 c \rho_{0}} u u_{\xi \xi}\right. \\
& \left.-\frac{\mu}{4 c \rho_{0}}\left(u_{\xi}\right)^{2}+\frac{\mu^{2}}{8 c \rho_{0}^{2}} u_{\xi \xi \xi}\right]+O\left(\epsilon^{2}\right) .
\end{aligned}
$$

In order to compare to Eq. (1), we first have to rewrite Eq. (52) in a nondimensional form. To this end, we nondimensionalize all variables according to

$$
u \rightarrow \frac{u}{c}, \quad \xi \rightarrow-\frac{\rho_{0}}{c \mu} \xi, \quad \tau \rightarrow \frac{\rho_{0}}{2 c^{2} \mu} \tau
$$

In terms of these new variables, the nondimensional version of Eq. (52) reads

$$
\begin{aligned}
u_{\tau}= & 2 u u_{\xi}+u_{\xi \xi}+\epsilon\left[-\frac{2 \rho_{0}}{A \gamma} u^{2} u_{\xi}-\frac{3 \rho_{0}}{A \gamma} u u_{\xi \xi}\right. \\
& \left.-\frac{\rho_{0}}{2 A \gamma}\left(u_{\xi}\right)^{2}-\frac{\rho_{0}}{4 A \gamma} u_{\xi \xi \xi}\right]+O\left(\epsilon^{2}\right) .
\end{aligned}
$$

A comparison with Eq. (1) yields

$$
\begin{aligned}
& \alpha_{1}=-\frac{2 \rho_{0}}{3 A \gamma}, \quad \alpha_{2}=-\frac{\rho_{0}}{A \gamma}, \\
& \alpha_{3}=-\frac{\rho_{0}}{6 A \gamma}, \quad \alpha_{4}=-\frac{\rho_{0}}{4 A \gamma} .
\end{aligned}
$$

The linearizability condition (16), therefore, is

$$
\frac{3 \rho_{0}}{8 A \gamma}=0 .
$$

This means that, in the long-wavelength, small-amplitude limit, the equation governing a one-dimensional gas can be linearized only at the lowest order. When the $O(\epsilon)$ corrections are taken into account, the corresponding equation cannot be linearized, indicating that obstacles to linearizability are present already at this order.

\section{FINAL REMARK}

In the case of the traveling-wave solution to the Burgers equation, the above analysis simplifies considerably. As we have already shown, $Z(w)=0$ for the shock solution. In this case, however, we could have looked directly to Eq. (20) without need of a Hopf-Cole transformation. Indeed, if we take

$$
w=-k\left[1-\tanh \left(k x-2 k^{2} t\right)\right]
$$

which is a solution of the Burgers equation, we can promptly verify that $\nu_{1}=-\nu_{2}=-\nu_{3}=-\frac{2}{3}$ implies $Z(w)=0$. This makes it easy to find the $O(\epsilon)$ correction to the solution (57) coming from the joint solution satisfying simultaneously the Burgers and the first higher-order equation of the Burgers hierarchy, which can be verified to be [13]

$$
w=-k\left\{1-\tanh \left[k x-\left(2 k^{2}-4 \epsilon \alpha_{4} k^{3}\right) t\right]\right\} .
$$

Finally, we note that, having obtained the function $w(x, t)$, the physical variable $u(x, t)$ is obtained through Eq. (3). In the case under examination, using the fact that $\alpha$ has been left arbitrary, we come to the following form for $\phi(w)$, which represents the effects of the perturbation terms present in Eq. (1):

$$
\begin{aligned}
\phi(x, t)= & -2 k^{2} \beta \tanh (\bar{\Theta})+\gamma k^{2} \operatorname{sech}^{2}(\bar{\Theta}) \\
& \times\left\{\ln \left[\frac{1}{2}\left(1+e^{-2 \bar{\Theta}}\right)\right]\right\},
\end{aligned}
$$

where $\bar{\Theta}=k x-\left(2 k^{2}-4 \epsilon \alpha_{4} k^{3}\right) t$. The first term represents a modification of amplitude of the shock solution. The second term represents a deformation profile. Notice, however, that 
this second term is important only in the region near the origin of the $\bar{\Theta}$ axis, falling to zero as $\bar{\Theta}$ increases. The relative importance of the deformation with respect to the amplitude modifications depends moreover on the coefficients $\beta$ and $\gamma$, which in turn depend on the coefficients of Eq. (1).

Recently, we came to know that part of our results con- cerning the existence of near-identity transformations had been independently obtained in Ref. [14].

\section{ACKNOWLEDGMENT}

The authors would like to thank CNPq, Brazil, for partial financial support.
[1] R. K. Dodd, J. C. Eilbeck, J. D. Gibbon, and H. C. Morris, Solitons and Nonlinear Wave Equations (Academic, London, 1982).

[2] R. A. Kraenkel, J. G. Pereira, and M. A. Manna, Phys. Rev. A 45, 838 (1992).

[3] F. Calogero, Why Are Certain Nonlinear PDEs Both Widely Applicable and Integrable, in What is Integrability, edited by V. E. Zakharov (Springer, Berlin, 1991).

[4] G. B. Whitham, Linear and Nonlinear Waves (Wiley, New York, 1973).

[5] D. V. Choodnovsky and G. V. Choodnovsky, Nuovo Cimento B 40, 339 (1977).

[6] V. I. Arnold, Geometrical Methods in the Theory of Ordinary Differential Equations (Springer, Berlin, 1983).
[7] Y. Kodama, Phys. Lett. 112A, 193 (1985).

[8] A. S. Fokas and Q. M. Liu, Phys. Rev. Lett. 77, 2347 (1996).

[9] Y. Kodama, Phys. Lett. A 123, 276 (1987).

[10] R. A. Kraenkel, M. A. Manna, V. Merle, J. C. Montero, and J. G. Pereira, Phys. Rev. E 54, 2976 (1996).

[11] Y. Kodama and A. V. Mikhailov, Obstacles to Asymptotic Integrability, in Algebraic Aspects of Integrable Systems: In Memory of Irene Dorfman, edited by A. S. Fokas and I. M. Gelfand (Birkhäuser, Boston, 1996).

[12] J. Kevorkian and J. D. Cole, Multiple Scale and Singular Perturbation Methods (Springer, New York, 1996).

[13] Y. Kodama and T. Taniuti, J. Phys. Soc. Jpn. 47, 1706 (1979).

[14] T. Fokas and L. Luo, Contemp. Math. 200, 85 (1996). 